\title{
Does COVID-19 affect food safety and security?
}

\author{
A summary report on the Extraordinary Scientific Roundtable \\ of IUFoST-CIFST on March 21, 2020
}

\begin{abstract}
The goal of this contribution is to provide a summary report on the Extraordinary Scientific Roundtable on COVID-19 and Food Safety co-organized by the International Union of Food Science and Technology (IUFoST) and the Chinese Institute of Food Science and Technology (CIFST). The meeting provided a valuable recount of this coronavirus, particularly that the virus is not transmitted by food. On this basis, so far there is no evidence that COVID-19 has any effect on food safety and security as well as on food bioactives. The challenges faced by the food industry during the pandemic period as well as potential post-pandemic time opportunities were discussed.
\end{abstract}

This brief write-up takes advantage of a recent Extraordinary Scientific Roundtable held on March 21, 2020 under the auspices of the International Union of Food Science and Technology (IUFoST), which is the global scientific organization representing national societies and institutes from many countries with membership of food scientists, technologists and engineers. The roundtable, which was convened in cooperation with the Chinese Institute of Food Science and Technology (CIFST) included a number of scientists from international and national emergency response teams as well as a representative of the food industry. The meeting was chaired by Dr. Fereidoon Shahidi who is the Chair of the Scientific Council of IUFoST and co-chaired by Dr. Junshi Chen, Chief Adviser, China National Center for Food Safety Risk Assessment and Dr. Pingfan Rao, IUFoST Past President and Professor and founding Director of CAS.SIBS-Zhejiang Gongshang University Joint Center for Food and Nutrition Research in Hangzhou, China. The panel members were Dr. Peter Ben Embarek, Unit Head, Monitoring of Nutrition and Food Safety Events, International Food Safety Authorities Network (INFOSAN), World Health Organization (WHO), Dr. Ding Gangqiang, Vice President of CIFST and Director of Nutrition and Health Department within the China Centers of Disease Control, who was deployed in Wuhan, Dr. Patrick Wall, member of the Ireland COVID-19 National Emergency Response and Professor of Public Health at University College, Dublin, Dr. Luo Yunbo, Honorary Vice President of CIFST and Director of the Research Centre for Special Food, China Agricultural University, and Ms. Liz Duffy, Vice President for Omnichannel Compliance for Walmart Global eCommerce.

The WHO has stated that COVID-19 appeared to be a zoonotic virus originating in bats but is likely to have gone through an intermediate species to infect humans. Zoonotic diseases are infectious ailments caused by bacteria, viruses and parasites transmitted directly from animals to humans. Some $75 \%$ of emerging infectious agents are zoonotic. The importance of controlling zoonotic diseases was emphasized by WHO beginning with the One Health initiative, although most of these diseases are not prioritized by health systems internationally or by most countries. The experience of China to bring the COVID-19 a successful level of control provides a message of hope for the rest of the world, showing that this outbreak can be brought under control even though the economic and social costs are high. Several other countries, notably South Korea and Singapore, have also shown that it can be controlled if the right strategies are devised.

It is important to note that there have been no reports of transmission of COVID-19 through food. Therefore, based on current epidemiological support, this virus is not foodborne. It was noted that the United States Centre for Disease Control and European Centre for Disease Control have stated that there is also no evidence that food items imported from China, in accordance with related regulations, will pose any risk of spreading COVID-19 in their countries. In addition, it was emphasized that since, in general, no viable viruses survive heat treatment, all cooked food should be safe to eat. However, it is most important to refrain from consuming raw or undercooked animal products.

The WHO has also stated that the COVID-19 virus is transmitted through droplets, or small bits of liquid, primarily through sneezing and coughing that produce $3,000-10,000$ droplets that cause "droplet spread". This conclusion has been supported by other food safety and health authorities. The possible presence of the virus on food packaging material, as such, is not currently considered to be causing infection. It might, however, be a function of the pore size of the material, which has not yet been fully evaluated (this is a personal opinion of the author and not expressed by members of the panel). The panel members stated that the effective methodology used in Wuhan to control the disease should be employed as much as possible, namely, to identify, admit and isolate confirmed COVID-19 cases and to follow up with all their known contacts. In such cases, it is necessary to 
have adequate capacity to isolate and treat severe cases. Here, it is again the personal view of the author that, in the case of the fight against this virus, personal freedoms must take a lower priority compared to the welfare of society at large. In the case of Wuhan, hotels were used to isolate people who were at risk of infecting others. The nutritional impact of isolation of individuals indicated that while their energy intake remained unchanged, their intake of vitamins, minerals and plant-based protein and fatty acids was compromised. In addition, the lack of physical activity may cause obesity and non-communicable diseases (NCD) in the long term. Thus, the elderly population needs to pay greater attention to dietrelated and other risk factors. In Wuhan, providing safe and nutritionally balanced meals to doctors, nurses and other healthcare professionals and also ambulance drivers were considered essential. Cooked meals were continuously made available to them in the areas they were serving during the crisis in spite of the difficulties encountered.

With respect to food availability, it was noted that primary production may not be severely affected because production areas are often geographically distant from urban population densities. However, the pandemic may affect harvesting, transportation and distribution. In this regard, animal feed and ingredients for food product preparation may be adversely affected, especially if they need to be imported. This is particularly true as many of the primary ingredients originate from China and India for both food and non-food items, such as pharmaceuticals. Thus, the present reliance on specific markets, products and distribution channels is probably insufficient or inadequate to respond to anticipated disruptions cause by the pandemic. In order to fight this virus, travel, conferences, conventions and sporting events have been cancelled and food service providers as well as the entire tourism industry have been decimated due to the required social distancing. All of these have consequences for human resources and the economy. The food industry needs to keep its staff healthy if it is to function successfully. Consumers may also resort to over-stocking and hoarding, which adversely affects the supply chain. This happens mostly due to believing rumors and false information from unauthorized sources.

In order to keep our food supply safe, it is imperative that the real importance of food safety with respect to good hygiene practices by all be emphasized. This is not just restricted to the food industry and must be practiced by everyone, including intermediaries from the food industry to the consumer. All must be reminded to follow the WHO Five Keys to Safer Food (https:// www.who.int/foodsafety/areas_work/foodhygiene/en/), particularly handwashing. The disruption of the food supply chain may also create unexpected food safety risks, particularly for perishable foods and vegetables. These have often been a source of concern due to inadequate washing and packaging. It is also necessary to communicate food safety information in a simple and understandable manner. This should be done in a scientifically sound and thoughtful way with respect to those involved in the food chain, from producers to intermediaries and consumers, particularly in these uncertain times. Therefore, the development of safe and effective foods to promote immune function should be a priority for the food industry and governments. This is a new role for functional foods and may include special medical foods for the elderly as well as immune-compromised individuals and groups.

In view of the popularity of e-commerce in food, especially during the current situation with lockdowns and social distancing in effect, tremendous growth opportunities exist for the industry to cater and respond to the needs of consumers who do not want to shop in person. Thus, in the e-commerce space, in particular, consumers will solely or primarily shop online and may spend a significant amount of time online. They are getting inundated with information around the outbreak and unfortunately, a lot of that information is not founded on science. They are looking at social media sites and other information sources to gain knowledge about the outbreak, and so it is important that consumers get the most accurate and scientifically-based information from academic, industry and government sources.

What is evident to all, and was agreed by the panel members, is the necessity to educate consumers and provide them with accurate information so that they can make sound decisions. This must be done in a timely manner as we are dealing with a rapidly evolving situation. The food industry must ensure the availability of safe food with adequate wholesomeness and nutritional attributes to the global community, especially because certain raw materials and ingredients may need to be substituted and processing conditions changed or modified. This is particularly essential for large cities and urban populations in developing countries. Meanwhile, it is essential that the personnel working in the food industry and allied areas of food supply chain remain in good health with consideration of physical distancing in processing and preparation plants. This might be provided by temporary zoning, which may not be possible in all cases. However, where such an option exists, only a small number of people are affected and removed if a member tests positive for the virus. Personnel who contract the virus must be given the ability to immediately report it to their supervisors and remain isolated at home. This may result in labor shortages and arrangements should be in place by all means, including e-technology, to recruit and train new staff. Workers from commercial catering, tourism and hospitality industries with food experience can be redeployed into other parts of the food industry. The food industry needs to address the anxiety of the consumers via clear, science-based and user-friendly messages that do not make any false claims. The current food safety protocols, including food recalls, must be put in place, if and when needed.

It was also noted that the post-pandemic phase may result in major reviews of food systems with special emphasis on resilience. Thus, the agri-food systems may take advantage of the opportunities to make changes, such as making more use of locally produced food. This would remove dependence on long-distance transportation and distribution by third parties with major carbon footprints. A paradigm shift for safe food practices should be reinforced by promoting safety habits that were developed and acquired during the pandemic. The food science and technology community will be in a position to strategically plan and contribute to the recovery of the food sector in collaboration with other allied disciplines and stakeholders. The role of food scientists and technologists in shaping the policies of governments and in decision-making strategies must be considered in order to ensure the readiness of the food supply chain in responding to any future pandemic.

In summary, it was mentioned that the pandemic of COVID-19 posed unprecedented challenges to the food sector, especially when many people may need to be quarantined for at least a twoweek period. However, it was emphasized that the food industry with the help of food scientists and technologists can overcome these challenges and succeed in providing safe, nutritious and sufficient food to the global population. In the future, emphasis may also shift to providing immune-enhancing functional foods, which indicates the important role of food bioactive compounds in reducing disease risk.

Finally, it is important to emphasize here the commitment of IUFoST and its national affiliates in assisting the food industry in its essential role in society. The International Society for Nutraceu- 
ticals and Functional Foods (ISNFF) www.isnff.org, a disciplinary interest group of the IUFoST, and its current main publication, the Journal of Food Bioactives (JFB) www.isnff-jfb.com are proud to take all measures needed to be a part of this effort to fulfilling its workplan with regard to addressing food safety and security as well as food supply problems in the developing countries. In this regard, a Task Force was established by the IUFoST to share the findings and experiences of the food industry in one place with all. For further information, interested parties may contact secretariat@iufost.org or the author at fshahidi@gmail.com.

Fereidoon Shahidi

Department of Biochemistry, Memorial University of Newfoundland, St. John's, NL, Canada A1B 3X9 